\title{
Role of Two Dimension Strain and Strain Rate Echocardiography in Assessment of Rheumatic Mitral Valve Stenosis
}

\author{
Wassam ElDin Hadad ELShafey, Walaa Farid, Sara Abd El Maksoud \\ Menoufia University, Al Minufya, Egypt \\ Email: dr_wesamhadad@yahoo.com
}

How to cite this paper: ELShafey, W.E.H., Farid, W. and El Maksoud, S.A. (2019) Role of Two Dimension Strain and Strain Rate Echocardiography in Assessment of Rheumatic Mitral Valve Stenosis. World Journal of Cardiovascular Diseases, 9, 759-771. https://doi.org/10.4236/wjcd.2019.910068

Received: September 27, 2019

Accepted: October 20, 2019

Published: October 23, 2019

Copyright () 2019 by author(s) and Scientific Research Publishing Inc. This work is licensed under the Creative Commons Attribution International License (CC BY 4.0).

http://creativecommons.org/licenses/by/4.0/

\begin{abstract}
Introduction: Current echocardiographic parameters have a limitation in assessing mitral valve (MV) apparatus in rheumatic mitral stenosis (MS) patient. In the current study, we use 2 dimensional (2D) longitudinal strain (S) and strain rate ( $\mathrm{Sr}$ ) imaging in evaluating the papillary muscle longitudinal strain (LS) as an objective and quantitative echocardiographic parameter with high reproducibility in the assessment of MV apparatus in patients with mild to moderate rheumatic MS with preserved ejection fraction (EF\%). Patients and Method: The study included 40 patients with established diagnosis of MS subdivided in to 31 patients with moderate MS (mean age: $32 \pm 5$ ) and 9 patients with mild MS (mean age: $31 \pm 6$ ). 20 healthy individuals (mean age $31 \pm 6)$ as a control for cases. The mitral valve area (MVA) was estimated using planimetry and pressure half time (PHT) methods. 2D longitudinal systolic $\mathrm{S}$ and $\mathrm{Sr}$ imaging was carried out for all participants from the apical long axis (LAX), 4 chamber (4C), 2 chamber (2C) views. Global longitudinal systolic $\mathrm{S}$ and $\mathrm{Sr}$ were estimated by averaging the 3 apical views. Longitudinal myocardial strain of papillary muscle PMs was assessed by the use of the free strain method from apical 4 chamber view for the antrolateral papillary muscle (APM) and apical long axis view for postromedial papillary muscle (PPM). Results: Patients with MS had significantly decreased longitudinal LV systolic $\mathrm{S}$ and $\mathrm{Sr}$ in comparison with control group $(\mathrm{p}<0.001)$ despite no significant differences in LV EF\%, LVESD and LVEDD were determined between the 3 groups. APM-LS and PPM-LS had significantly decreased values in patient with MS in comparison with control group $(\mathrm{p}<0.001)$. Conclusion: Patients with MS and preserved EF\% had decreased APM-LS \& PPM-LS in comparison with control group, and had decreased longitudinal LV systolic $\mathrm{S}$ and $\mathrm{Sr}$ when compared with control group. 2D strain as well as Sr imaging might be a useful method for assessment of mitral valve apparatus in patients with MS \& preserved $\mathrm{EF} \%$.
\end{abstract}




\section{Keywords}

Strain, Strain Rate, LV Systolic Function, Papillary Muscle, Mitral Stenosis

\section{Introduction}

Indeed, the commonest pathophysiological etiology of MS is rheumatic heart disease [1]. The most commonly affected valve is MV, and it is markedly influenced where $65 \%-70 \%$ of rheumatic patients suffer from stenosis and/or regurgitation [2]. Chronic affection of the MV leaflets in rheumatic patients in particular with recurrent episodes includes chronic scarring [3].

These valvular changes become progressive over time resulting in funnelshaped stenotic MV. Rheumatic affection of subvalvular apparatus might be in the form of fusion, retraction, thickening, shortening, as well as calcification [3]. In MS, if it is considered that fibrosis mainly involves the valve along with nearby structures such as the papillary muscles APM \& PPM, the measurement of longitudinal strain of papillary muscles in addition to the underlying myocardium by $2 \mathrm{D}$ strain and $\mathrm{Sr}$ is new quantitative method for assessment of MV apparatus.

In fact, echocardiography is considered the primary diagnostic modality for evaluation of MV.

With implementing 2D strain and Sr echocardiography acquire an essential role as a technique that can assess the geometry and function of myocardium in MS.

Strain (S) is a measure of fiber shortening, and strain rate ( $\mathrm{Sr}$ ) assesses the deformation rate. Thus, both of two methods can give complementary information regarding the global \& regional myocardial function [4].

\section{Aim of the Work}

To assess MV apparatus along with LV systolic function in patients had mild to moderate MS with preserved EF\% via the use of 2D longitudinal systolic strain S and Sr imaging.

\section{Patients and Methods}

60 cases were rolled out in this study where 40 patients with established diagnosis subdivided into 2 subgroups 9 patients with mild MS, 31 patients with moderate MS and 20 subjects as a control. The study was approved by the ethics committee of the hospital and all included subjects have consented to participate in this study.

\section{Exclusion criteria}

1) Patients suffering from coronary artery disease (CAD) and apparent LV wall motion abnormalities.

2) Patients suffering from LV systolic dysfunction (EF\% less than 50\%). 
3) Patients suffering from cardiac rhythm or conduction disturbances as atrial fibrillation or artificial pacing.

4) Patients suffering from concomitant moderate or severe mitral regurgitation, aortic stenosis and aortic regurgitation.

Each person included in the study was subjected to:

1) Careful history taking and thorough physical examination.

2) Standard 12-lead electrocardiogram (ECG): For the assessment of cardiac rhythm, features suggesting chamber enlargement, and CAD.

\section{3) Conventional echocardiographic measurement.}

Patients were monitored through a single-lead ECG.

The left atrial diameter, LV end-diastolic \& end-systolic diameters, LV fractional shortening \%, the interventricular septum (IVS) thickness, and the posterior wall (PW) thickness were measured based on the American Society of Echocardiography recommendations [5].

The LV EF was calculated using modified Simpson's biplane method of disks. Traditional MS indices as maximum MV pressure gradient (PG) and mean MV pressure gradient (MG) were estimated. Mitral valve area (MVA) was measured using mitral orifice planimetry in parasternal short axis view, along with the Doppler derived pressure halftime method ( $\mathrm{PHT}$ ) and the average area was estimated using the mean value of 2 measurements. MS severity was measured according to hemodynamic data, using MVA, MG and pulmonary artery systolic pressure (PAP) as follows: mild MS (MVA more than $1.5 \mathrm{~cm}^{2}$, MG less than 5 $\mathrm{mmHg}$, or PAP less than $30 \mathrm{mmHg}$ ), moderate MS (MVA $1.0-1.5 \mathrm{~cm}^{2}$, MG 5 $10 \mathrm{mmHg}$, or PAP 30 - $50 \mathrm{mmHg}$ ), and severe MS (MVA less than $1.0 \mathrm{~cm}^{2}, \mathrm{MG}$ more than $10 \mathrm{mmHg}$, or PAP > $50 \mathrm{mmHg}$ ). PAP was calculated by adding 10 $\mathrm{mmHg}$, considering the diameter of the inferior vena cava (IVC) and level of its collapse that result due to respiration, to the value estimated by evaluating the Bernoulli equation that is simplified from tricuspid insufficiency velocities. The valvular incompetence was assessed by colour flow Doppler imaging.

4) Measurement of the 2D S and Sr: 2D echocardiography images (transmit/receive 1.9/4.0 $\mathrm{MHz}$ ) were obtained from LV apical long axis, 4 chamber, and 2 chamber views with frame rates of 50 - 90 frames/s. Digital data were stored and analyzed off-line. LV endocardial surface was traced manually, and the speckle tracking width was modified in order to cover the whole LV wall thickness so as to obtain curves. Peak LV longitudinal systolic strain (LSS) and Sr (LSSr) were calculated for apical LAX, 4C, and 2C views, and global LV systolic strain (GLSS) and Sr (GLSSr) were calculated through averaging the 3 apical views, Longitudinal myocardial strain of PMs was assessed by the free strain method from apical 4chamber view for APM and apical long axis view for PPM [6].

Patients in whom PM views were clearly visualized in both systole \&diastole were considered eligible for the assessment.

In fact, free strain method allows for the measuring of user defined custom local velocities, displacement \& deformation using unlimited directional chords 
display technique. This work flow measures strain within the myocardial region, free of restraints on the location or direction of the measurements that might be radial, longitudinal or circumferential. Free strain has the advantage of being easy, rapid and practical technique for measurement of myocardial deformation. This method is especially preferred in the measurement of PMs deformation as these structures are relatively separated from the LV myocardium and aren't included in the commercially available LV strain models [6].

Measurement of the longitudinal strain using the free strain method needs selection of the region of interest by clicking 2 points manually. The $1^{\text {st }}$ point was chosen from the base of the PM at its attachment zone to the LV wall. The $2^{\text {nd }}$ one was chosen from the tip of the PM with special attention so as to keep a 3 - 5 $\mathrm{mm}$ distance from the chordae aiming to avoid artifacts.

All STE acquisitions were carried out at frame rates between $50-70 \mathrm{~Hz}$ frames/second. The average value of $S$ was taken from the 3 consecutive beats. The peak systolic values were recorded for GCS, GLS and longitudinal S of APM \& PPM [6].

All the echocardiographic studies were done by one echocardiographer and for intra-observer variability, a sample of $2 \mathrm{D} \mathrm{S}$ and Sr measurements was randomly selected and examined by the same observer in 2 different days and intra-class correlation coefficients were calculated for the same observer.

\section{Statistical Analysis}

Data were fed to the computer and analyzed using IBM SPSS software package version 20.0. (Armonk, NY: IBM Corp) Qualitative data were described using number and percent. The Kolmogorov-Smirnov test was used to verify the normality of distribution Quantitative data were described using range (minimum and maximum), mean, standard deviation, median and interquartile range (IQR). Significance of the obtained results was judged at the $5 \%$ level.

The used tests were:

\section{1) Chi-square test}

For categorical variables, to compare between different groups

\section{2) Student t-test}

For normally distributed quantitative variables, to compare between two studied groups

\section{3) Pearson coefficient}

To correlate between two normally distributed quantitative variables

\section{4) F-test (ANOVA)}

For normally distributed quantitative variables, to compare between more than two groups, and Post Hoc test (Tukey) for pairwise comparisons

\section{5) Kruskal Wallis test}

For abnormally distributed quantitative variables, to compare between more than two studied groups, and Post Hoc (Dunn's multiple comparisons test) for pairwise comparisons. 


\section{6) Receiver operating characteristic curve (ROC)}

It is generated by plotting sensitivity (TP) on Y axis versus 1-specificity (FP) on $\mathrm{X}$ axis at different cut off values. The area under the ROC curve denotes the diagnostic performance of the test. Area more than 50\% gives acceptable performance and area about $100 \%$ is the best performance for the test. The ROC curve allows also a comparison of performance between two tests.

\section{7) Sensitivity}

The capacity of the test to correctly identify diseased individuals in a population "TRUE POSITIVES". The greater the sensitivity, the smaller the number of unidentified case "false negatives".

\section{8) Specificity}

The capacity of the test to correctly exclude individuals who are free of the disease "TRUE NEGATIVES". The greater the specificity, the fewer "false positives" will be included.

\section{9) Positive Predictive value (PPV)}

The probability of the disease being present, among those with positive diagnostic test results.

\section{0) Negative Predictive value (NPV)}

The probability that the disease was absent, among those whose diagnostic test results were negative.

\section{1) Accuracy}

Rate of Agreement $=($ True positives + True negatives $) /$ Total tested $\times 100$.

\section{Results}

\subsection{Demographic and Conventional Echocardiographic Characteristic}

Age \& gender indices there was no significance difference between the control group and patients with MS ( $p>0.05)$, heart rate was significantly increased in patients with MS in comparison with the control group $(\mathrm{p}<0.001)$.

There was no significant difference between systolic and diastolic blood pressures in patients with MS in comparison with the control group ( $p>0.05)$. No significant difference was observed in IVS and PW thickness, LVESD, LVEDD, $\mathrm{EF} \%$ and FS\% in patients with MS when compared with the control group ( $\mathrm{p}>$ $0.05)$, LA diameter was significantly higher in patients with MS in comparison with the control group $(<0.001)$. Transmitral PG, MG and mean MVA was significantly increased in cases with moderate MS than mild MS $(\mathrm{P}<0.001)$ (Table $1)$.

\subsection{D S and Sr Measurements}

There was a highly significant lower peak LV longitudinal systolic strain (LAXLSS, 4C-LSS, 2C-LSS and GLSS) in patients with MS in comparison with the control group $(<0.001)$.

A significantly lower peak LV longitudinal systolic strain rate (LAX-LSSr, 
Table 1. Comparison between the different studied groups according to different parameters.

\begin{tabular}{|c|c|c|c|c|c|}
\hline & \multirow[b]{2}{*}{$\begin{array}{l}\text { Control } \\
(n=20)\end{array}$} & \multicolumn{2}{|c|}{ Cases } & \multirow[b]{2}{*}{$\mathrm{p}$} & \multirow[b]{2}{*}{ Significance } \\
\hline & & $\begin{array}{l}\text { Mild MS } \\
(n=9)\end{array}$ & $\begin{array}{c}\text { Moderate MS } \\
\quad(\mathrm{n}=31)\end{array}$ & & \\
\hline Male/Female & $7 / 13$ & $4 / 5$ & $10 / 21$ & 0.796 & NS \\
\hline Age (years) & $31.55 \pm 5.56$ & $31.67 \pm 5.36$ & $32.26 \pm 4.92$ & 0.881 & NS \\
\hline Heart rate (bpm) & $66.45 \pm 4.29$ & $75.11^{\mathrm{a}} \pm 6.53$ & $75.39^{\mathrm{a}} \pm 5.86$ & $<0.001^{*}$ & $S$ \\
\hline Systolic BP (mmHg) & $107.90 \pm 10.25$ & $109.44 \pm 7.94$ & $107.35 \pm 9.29$ & 0.843 & NS \\
\hline Diastolic BP (mmHg) & $66.95 \pm 8.09$ & $66.22 \pm 2.54$ & $66.0 \pm 5.16$ & 0.860 & NS \\
\hline IVS thickness (mm) & $9.15 \pm 1.31$ & $9.56 \pm 1.13$ & $9.26 \pm 1.48$ & 0.765 & NS \\
\hline PWT (mm) & $9.90 \pm 1.02$ & $10.56 \pm 1.51$ & $10.26 \pm 0.77$ & 0.223 & NS \\
\hline LA diameter (mm) & $33.10 \pm 3.39$ & $42.0^{\mathrm{a}} \pm 1.32$ & $44.13^{\mathrm{a}} \pm 1.98$ & $<0.001^{*}$ & $S$ \\
\hline $\begin{array}{l}\text { LV end-Diastolic } \\
\text { diameter }(\mathrm{mm})\end{array}$ & $48.45 \pm 2.65$ & $47.67 \pm 1.87$ & $47.16 \pm 2.49$ & 0.245 & NS \\
\hline $\begin{array}{l}\text { LV end-systolic } \\
\text { diameter (mm) }\end{array}$ & $33.35 \pm 2.35$ & $31.89 \pm 3.41$ & $32.65 \pm 3.28$ & 0.464 & NS \\
\hline FS (\%) & $31.20 \pm 3.35$ & $31.56 \pm 6.04$ & $30.52 \pm 4.79$ & 0.784 & NS \\
\hline $\mathrm{EF}(\%)$ & $62.90 \pm 5.37$ & $59.78 \pm 8.47$ & $59.10 \pm 7.15$ & 0.153 & NS \\
\hline MVA $\left(\mathrm{cm}^{2}\right)$ & - & $1.61 \pm 0.08$ & $1.33 \pm 0.13$ & $<0.001^{*}$ & $s$ \\
\hline MG (mmHg) & - & $3.87 \pm 0.21$ & $6.81 \pm 1.44$ & $<0.001^{*}$ & $S$ \\
\hline PG (mmHg) & - & $8.67 \pm 0.50$ & $14.71 \pm 2.70$ & $<0.001^{*}$ & $S$ \\
\hline
\end{tabular}

Abbreviations: SBP, systolic blood pressure; DBP, diastolic blood pressure; IVS, interventricular septum; PWT, posterior wall thickness; LV, left ventricle; EF; ejection fraction, FS, fractional shortening; MVA, mitral valve area; PG, transmitral peak gradient; MG, mean gradient.

4C-LSSr, 2C-LSSr and GLSSr) was determined in patients with MS when compared with the control group $(<0.001)$, also there was significantly decreased papillary muscle longitudinal strain (APM-LS) \& (PPM-LS) in patients with MS in comparison with the control group $(\mathrm{p}<0.001)$ as shown in Table 2.

- Pearson correlation coefficient revealed a positive and significant correlation between LV GLSS and MVA $(r=0.838, \mathrm{p} \leq 0.001)$, APM-LS and MVA $(\mathrm{r}=$ $0.893, p=0.001)$, PPM-LS and MVA $(r=0.808, p \leq 0.001)$, Negative significant correlation between GLSS and MG $(\mathrm{r}=-0.789, \mathrm{p} \leq 0.001)$, APM-LS and MG $(r=-0.838, p \leq 0.001)$, PPM-LS and MG $(r=-0.751, p \leq 0.001)$ in patients with MS, as shown in Table 3.

- In order to evaluate the validity of the papillary muscles longitudinal strain (APM-LS \& PPM-LS) as a quantitative reproducible parameters to anticipate cases with moderate MS; APM-LS analysis showed a cut off value $\leq 16 \%$ sensitivity of $83.87 \%$ and a specificity of $100 \%$ with a positive predictive value (PPV) $100 \%$ and negative predictive value of $64.3 \%$. regarding PPM-LS; analysis exhibited a cut off value $\leq 15 \%$ sensitivity of $87.10 \%$ and a specificity of $88.89 \%$ with PPV $96.4 \%$ and negative predictive value of $66.7 \%$ (Table 4 , Figures 1-4). 
Table 2. Systolic strain and strain rate measurements of the study groups.

\begin{tabular}{|c|c|c|c|c|c|}
\hline & \multirow{2}{*}{$\begin{array}{l}\text { Control } \\
(n=20)\end{array}$} & \multicolumn{2}{|c|}{ Cases $(n=40)$} & \multirow[b]{2}{*}{$\mathrm{p}$} & \multirow[b]{2}{*}{ Significance } \\
\hline & & $\begin{array}{l}\text { Mild MS } \\
(\mathrm{n}=9)\end{array}$ & $\begin{array}{l}\text { Moderate MS } \\
\quad(\mathrm{n}=31)\end{array}$ & & \\
\hline LAX-LSS\% & $-20.20 \pm 1.94$ & $-17.0^{\mathrm{a}} \pm 1.22$ & $-14.19^{\mathrm{ab}} \pm 1.30$ & $<0.001^{*}$ & S \\
\hline 4C-LSS\% & $-20.55 \pm 1.57$ & $-17.11^{\mathrm{a}} \pm 1.36$ & $-14.35^{\mathrm{ab}} \pm 1.25$ & $<0.001^{*}$ & $S$ \\
\hline 2C-LSS\% & $-18.90 \pm 2.25$ & $-16.0 \pm 1.22$ & $-13.45^{\mathrm{ab}} \pm 1.18$ & $<0.001^{*}$ & $S$ \\
\hline GLSS\% & $-19.88 \pm 1.57$ & $-16.73^{\mathrm{a}} \pm 1.26$ & $-14.02^{\mathrm{ab}} \pm 1.23$ & $<0.001^{*}$ & s \\
\hline LAX-LSSr s-1 & $-1.38 \pm 0.14$ & $-1.09^{\mathrm{a}} \pm 0.02$ & $-1.05^{\mathrm{ab}} \pm 0.03$ & $<0.001^{*}$ & $S$ \\
\hline 4C-LSSr s-1 & $-1.40 \pm 0.14$ & $-1.10^{\mathrm{a}} \pm 0.03$ & $-1.09^{\mathrm{ab}} \pm 0.18$ & $<0.001^{*}$ & $S$ \\
\hline 2C-LSSr s-1 & $-1.37 \pm 0.14$ & $-1.08^{\mathrm{a}} \pm 0.02$ & $-1.04^{\mathrm{ab}} \pm 0.03$ & $<0.001^{*}$ & $S$ \\
\hline GLSSr s-1 & $-1.38 \pm 0.14$ & $-1.09^{\mathrm{a}} \pm 0.02$ & $-1.06^{\mathrm{ab}} \pm 0.06$ & $<0.001^{*}$ & $s$ \\
\hline APM-LS\% & $-24.35 \pm 1.42$ & $-18.44^{\mathrm{a}} \pm 1.13$ & $-15.10^{\mathrm{ab}} \pm 1.76$ & $<0.001^{*}$ & $s$ \\
\hline PPM-LS\% & $-23.05 \pm 1.32$ & $-16.44^{\mathrm{a}} \pm 0.88$ & $-14.03^{\mathrm{ab}} \pm 1.33$ & $<0.001^{*}$ & s \\
\hline
\end{tabular}

Abbreviations: LAX-LSS, apical long axis longitudinal systolic strain; 4C-LSS, apical 4 chamber longitudinal systolic strain; 2CLSS, apical 2 chamber longitudinal systolic strain; GLSS, left ventricular global longitudinal systolic strain; LAX-LSSr, apical long axis longitudinal systolic strain rate; 4C-LSSr, apical 4 chamber longitudinal systolic strain rate; 2C-LSSr, 2 chamber longitudinal systolic strain rate; GLSSr, left ventricular global longitudinal systolic strain rate, APM-LS, anterolateral papillary muscle longitudinal strain, PPM-LS, posteromedial papillary muscle longitudinal strain.

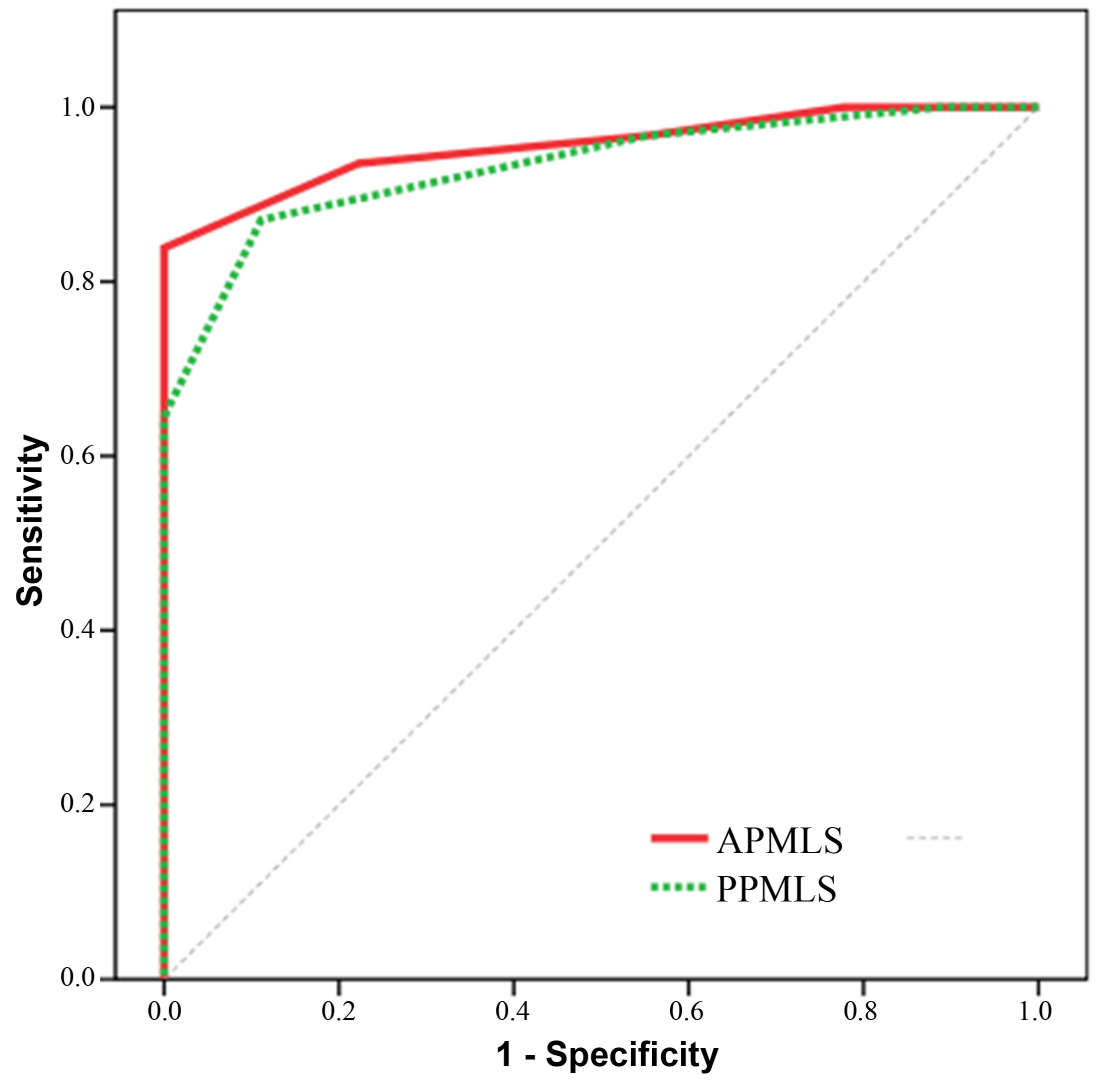

Figure 1. ROC curve for different parameters to predict cases with moderate MS for cases. 
Table 3. Correlation between MVA and MG with different parameters in each group $(\mathrm{n}=40)$.

\begin{tabular}{|c|c|c|c|c|c|c|c|c|c|c|c|c|}
\hline & \multicolumn{4}{|c|}{ Total cases $(n=40)$} & \multicolumn{4}{|c|}{ Mild MS $(n=9)$} & \multicolumn{4}{|c|}{ Moderate MS $(\mathrm{n}=31)$} \\
\hline & \multicolumn{2}{|c|}{ MVA } & \multicolumn{2}{|c|}{ MG } & \multicolumn{2}{|c|}{ MVA } & \multicolumn{2}{|c|}{ MG } & \multicolumn{2}{|c|}{ MVA } & \multicolumn{2}{|c|}{ MG } \\
\hline & $\mathbf{r}$ & $\mathrm{p}$ & $\mathbf{r}$ & $\mathrm{p}$ & $\mathbf{r}$ & $\mathrm{p}$ & $\mathbf{r}$ & $\mathrm{p}$ & $\mathbf{r}$ & $\mathrm{p}$ & $\mathbf{r}$ & $\mathrm{p}$ \\
\hline GLSS & $0.838^{*}$ & $<0.001^{*}$ & $-0.789^{\star}$ & $<0.001^{\star}$ & $0.669^{*}$ & $0.049^{*}$ & $-0.775^{\star}$ & $0.014^{*}$ & $0.706^{*}$ & $<0.001^{\star}$ & $-0.643^{\star}$ & $<0.001^{\star}$ \\
\hline APM - LS\% & $0.893^{*}$ & $<0.001^{\star}$ & $-0.838^{\star}$ & $<0.001^{\star}$ & 0.503 & 0.168 & -0.465 & 0.207 & $0.834^{\star}$ & $<0.001^{\star}$ & $-0.730^{\star}$ & $<0.001^{\star}$ \\
\hline PPM - LS\% & $0.808^{\star}$ & $<0.001^{\star}$ & $-0.751^{\star}$ & $<0.001^{\star}$ & 0.282 & 0.462 & -0.321 & 0.400 & $0.697^{\star}$ & $<0.001^{\star}$ & $-0.578^{\star}$ & $<0.001^{\star}$ \\
\hline
\end{tabular}

$\mathrm{r}$ : Pearson coefficient; ${ }^{*}$ : Statistically significant at $\mathrm{p} \leq 0.05$.

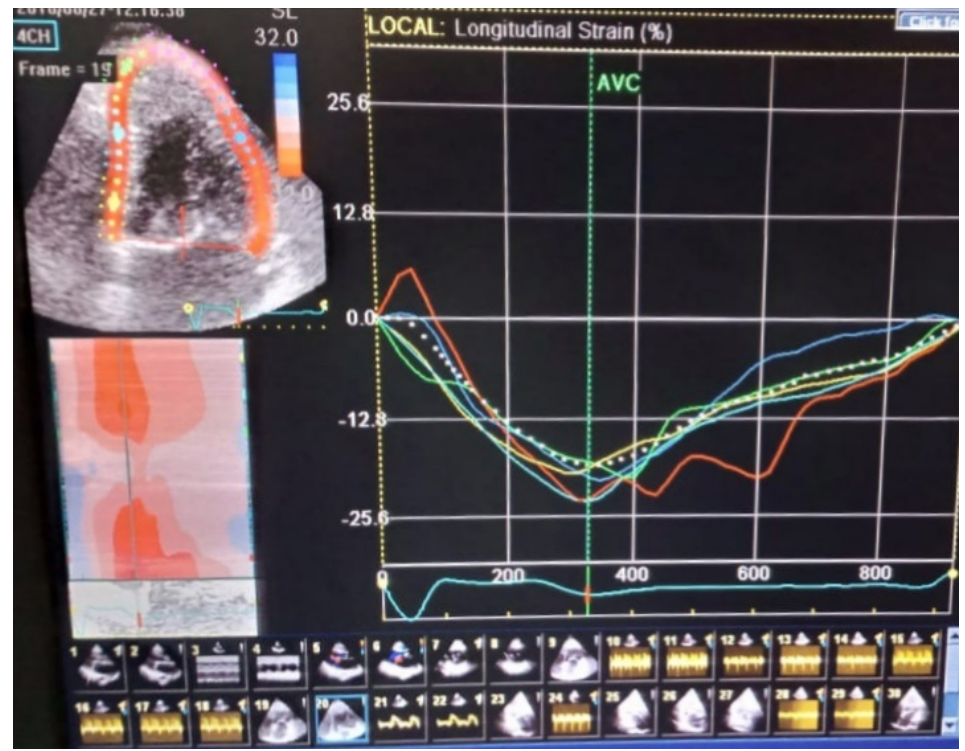

Figure 2. Shows apical $4 \mathrm{C}$ view with $2 \mathrm{D}$ strain of left ventricular septal and lateral wall with GS $=-19 \%$.

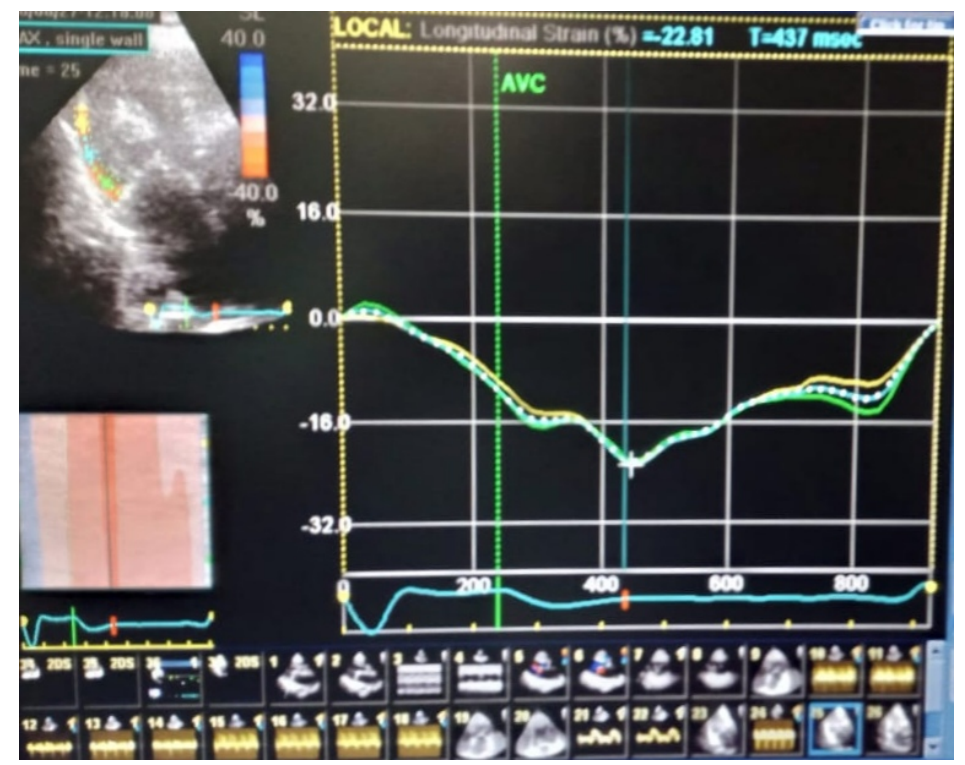

Figure 3. Shows apical parasternal long axis view with 2D strain of underlying LV inferior wall. 
Table 4. Agreement (sensitivity, specificity) for different parameters to predict cases with moderate MS for cases.

\begin{tabular}{ccccccccc}
\hline & AUC & p & $95 \%$ C.I & Cut off & Sensitivity & Specificity & PPV & NPV \\
\hline APM - LS\% & $0.955^{*}$ & $<0.001^{*}$ & $0.896-1.014$ & $\leq 16$ & 83.87 & 100.0 & 100.0 & 64.3 \\
PPM - LS\% & $0.932^{*}$ & $<0.001^{\star}$ & $0.854-1.010$ & $\leq 15$ & 87.10 & 88.89 & 96.4 & 66.7 \\
\hline
\end{tabular}

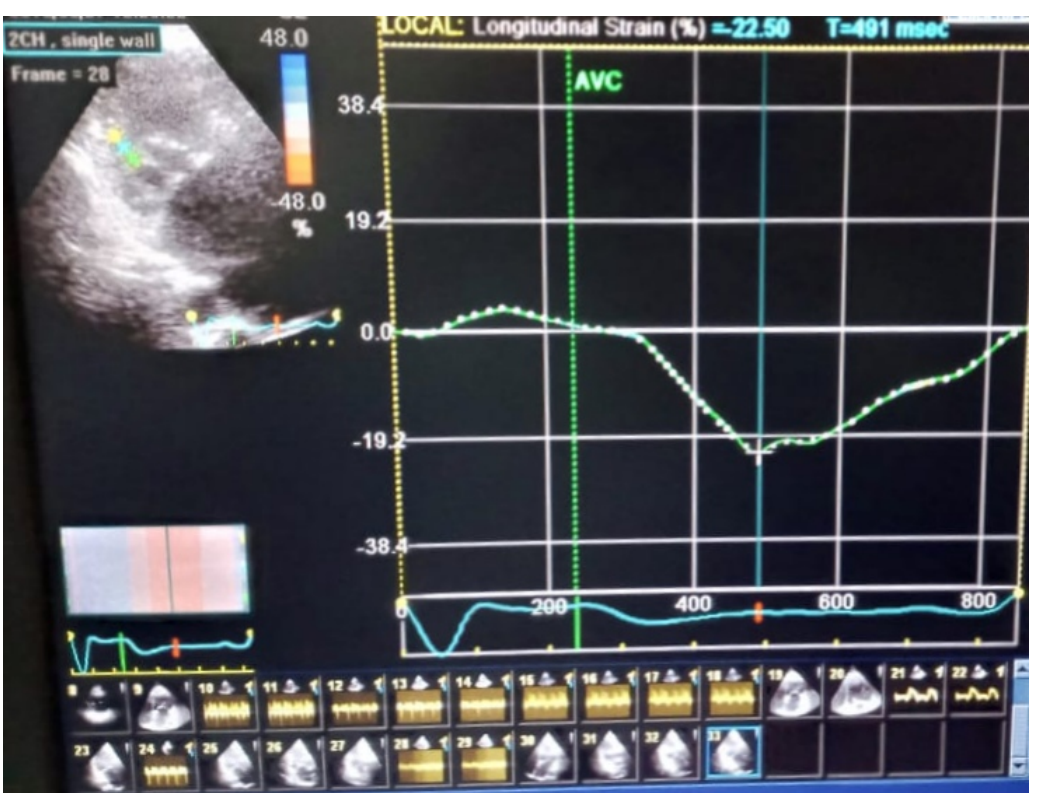

Figure 4. Shows apical parasternal long axis view with free $2 \mathrm{D}$ strain of postero-medial $\mathrm{PM}=-22 \%$.

\section{Discussion}

Rheumatic heart disease is the commonest pathophysiological etiology of MS. [1] indeed, the rheumatic fever incidence as well as its complications have decreased in developed countries; however, the disease is still considered a major health problem in the vast majority of developing countries. The most commonly \& severely affected valve is the MV where about $65 \%$ - $70 \%$ of patients affected by rheumatic process have stenosis and/or regurgitation [2]. Chronic rheumatic invovement of the MV leaflets mainly with recurrent episodes includes chronic scarring [3].

Over time, these changes become progressive leading to funnel-shaped stenotic MV. Rheumatic involvement of subvalvular apparatus might be in the form of fusion, thickening, retraction, shortening, and calcification. As a result, the free interchordal space decreases and the opened "leaflet-chordae tendineae tunnel" available for diastolic flow is reduced [3]. In MS, if it is considered that fibrosis mainly involves the valve and adjacent structures including the APM \& PPM, measuring the longitudinal strain of papillary muscles and the underlying myocardium by $2 \mathrm{D} \mathrm{S}$ and $\mathrm{Sr}$ is new quantitative method for the evaluation of mitral valve apparatus.

Echocardiography is considered the primary diagnostic modality for the eval- 
uation of MV. Applying the 2D S \& Sr makes echocardiography a pivotal technique for evaluation of the geometry as well as the function of myocardium in MS. Subclinical LV dysfunction caused by MS has been assessed by a lot of methods. Gash et al. [7] detected that ejection phase indices are reduced in patients with LV dysfunction in comparison with those who don't have LV dysfunction. Kurtulus et al. [8] used TDI, Dogan et al. [9] and Simsek et al. [10] used Doppler strain, and Ozdemir et al. [1] used 2D strain imaging for evaluating subclinical LV dysfunction in patients with MS. In almost all of these studies, mild \& moderate MS patients were included in the studies, whereas the number of severe MS patients was either very small or excluded.

However, to date, no enough data are available concerning the applicability of this technique for evaluation of MV apparatus in MS in particular APM \& PPM.

Strain is a measure of fiber shortening, and $\mathrm{Sr}$ measures the rate of deformation. Thus, both of these techniques can give complementary data regarding the global \& regional myocardial function [4]. TDI-derived strain imaging is highly angle dependent and it measures deformation in one dimension only. On the contrary, 2D strain analysis doesn't have angle limitations, permits deformation analysis in 2 dimensions, decrease intra-observer and inter-observer variability, and consume less time. So, 2D strain imaging can reflect subclinical heart disease, and true wall motion which can't be determined by using the TDI-derived strain analysis [11]. So, in the current study we used 2D S \& Sr imaging technique.

Regarding the longitudinal 2D strain we found that the global longitudinal systolic strain was significantly impaired in patients with MS compared with controls ( $\mathrm{P}$ value $\leq 0.001)$.

We measure the longitudinal strain of APM and PPM using the free strain method from apical 4C view for (APM) and apical long axis view for (PPM) [6]. It has been determined that the longitudinal strain of APM \& PPM was significantly reduced in patients with MS in comparison with normal controls (P value $\leq 0.001$ ).

Our results can be explained by long term sequelae of rheumatic fever, the endocardium and myocardium might be involved with variable degrees that leads to in the loss of normally functioning cardiomyocytes, cardiomyocytes necrosis and replacement by fibrosis \& calcification [1]. LV fibers at the subendocardial site are lined up longitudinally and intersects with the mitral annulus, also, the anterior mitral valve leaflet bound to the septum and the fibrous annulus of the heart, thus pathological events which involve the MV can affect the LV also [4].

By the use of electron beam microscopy Lee \& Lee [12] revealed variable degrees of ultra-structural pathological changes of LV muscle cells such as the myofibrils, mitochondria, nuclei and other sarcoplasmic elements.

Younan evaluated 50 patients with an established diagnosis of MS (mean age: $32 \pm 8$ years) and 30 age-matched healthy individuals (mean age $34 \pm 7$ years). He measured the MVA using planimetry and PHT methods. He performed 2D 
longitudinal systolic S \& Sr imaging for each participant from the apical long axis (LAX), 4C and 2C views. Global longitudinal systolic S and Sr were estimated via averaging the 3 apical views but he didn't assess the APM-LS \& PPM-LS.

He found that there was no significant differences in LV EF\%, LV end-systolic and end-diastolic dimensions between the 2 groups.

His study was in agreement our results where he found that patients with MS had significantly reduced 2D global longitudinal LV systolic strain (GLSS) $(12.77 \% \pm 1.4 \%)$ and GLSSr $(1.02 \pm 0.30)$ in comparison with the control group with GLSS of $(18.6 \% \pm 2.82 \%)$ and GLSSr $(1.25 \pm 0.34)$ (P value $<0.001$ and $<0.05$ respectively] [13].

APM-LS and PPM-LS were not included in his study whereas in our study we found significantly reduced APM-LS $(-15 \pm 1.76)$ for moderate MS cases and $(-18.44 \pm 1.13)$ for mild MS cases, significantly reduced PPM-LS $(-14.03 \pm 1.33)$ for moderate MS cases and $(-16.44 \pm 0.88)$ for mild MS cases when compared with control group $(-24.35 \pm 1.42)$ with $(\mathrm{P}$ value $\leq 0.001)$.

Yildirimtürk et al. found significantly reduction in the longitudinal and circumferential S \& Sr in patients with MS in comparison with healthy subjects ( $\mathrm{p}$ $<0.001$ ) [14]. They revealed decreased S \& Sr values in anterior septum and inferior walls and especially in basal segments in contrast to Özdemir et al. [1].

Yildirimtürk [14] was in agreement with our finding regarding global longitudinal systolic strain (GLSS) which is decreased in MS patients, but APM-LS and PPM-LS weren't included in his study.

Regarding Pearson correlation analysis of $S$ and traditional echocardiographic parameters, in our study, there was a positive and significant correlation between LV GLSS and MVA $(r=0.838, p \leq 0.001)$, APM-LS and MVA $(r=0.893$, $\mathrm{p} \leq 0.001)$, PPM-LS and MVA $(\mathrm{r}=0.808, \mathrm{p} \leq 0.001)$, Negative correlation but significant between GLSS \& MG $(r=-0.789, P \leq 0.001)$, APM-LS \& MG $(r=$ $-0.838, \mathrm{P} \leq 0.001)$, PPM-LS \& MG $(\mathrm{r}=-0.751, \mathrm{P} \leq 0.001)$ in total cases of MS. We divided total MS cases in to 2 subgroups mild MS and moderate MS.

In Ozdemir et al. study [1] neither longitudinal 2D strain nor $\mathrm{Sr}$ analysis were correlated with either MVA or transmitral diastolic pressures. He suggested that LV dysfunction in MS may result from the extension of the rheumatic process to the adjacent myocardial tissue and exhibit no correlation with the severity of MS.

Younan [13] found a significant positive correlation between LV GLSS with EF\% $(r=0.33, p=0.01) \&$ MVA $(r=0.40, p=0.001)$ and a significant negative correlation between LV GLSS with PG $(r=0.36, p=0.007)$ and MG $(r=0.40, p$ $=0.003)$ in patients with MS. In addition, there was a significant positive correlation between LV GLSSr with EF\% $(r=0.39, \mathrm{p}=0.02)$ and MVA $(\mathrm{r}=0.52, \mathrm{p}=$ $0.001)$ and a significant negative correlation between LV GLSSr with PG ( $\mathrm{r}=$ $0.43, \mathrm{p} 1=0.005)$ and MG $(\mathrm{r}=0.42, \mathrm{p}=0.006)$ in patients with MS.

\section{Conclusion}

Patients with MS and preserved EF\% had a decreased 2D longitudinal papillary 
muscles free strain APM-LS \& PPM-LS in comparison with the control group. Also they had a decreased 2D longitudinal LV systolic S \& Sr when compared to the control group. 2D S \& Sr imaging appears to be of value in the assessment of papillary muscles as a part of subvalvular apparatus and useful in determination of subclinical LV systolic dysfunction in patients with MS and preserved EF\%.

\section{Recommendation}

Assessing 2D free strain of papillary muscles of the MV could help as a quantitative parameter adding value to the traditional Wilkins score in accurately judging the choice of operative intervention.

\section{Conflicts of Interest}

The authors declare no conflicts of interest regarding the publication of this paper.

\section{References}

[1] Ozdemir, A.O., Kaya, C.T., Ozcan, O.U., Ozdol, C., Candemir, B., Turhan, S., et al. (2010) Prediction of Subclinical Left Ventricular Dysfunction with Longitudinal Two-Dimensional Strain and Strain Rate Imaging in Patients with Mitral Stenosis. The International Journal of Cardiovascular Imaging, 26, 397-404. https://doi.org/10.1007/s10554-009-9550-2

[2] Karthikeyan, G. and Mayosi, B.M. (2009) Is Primary Prevention of Rheumatic Fever the Missing Link in the Control of Rheumatic Heart Disease in Africa? Circulation, 120, 709-713. https://doi.org/10.1161/CIRCULATIONAHA.108.836510

[3] Kaplan, E.L. (2005) Pathogenesis of Acute Rheumatic Fever and Rheumatic Heart Disease: Evasive after Half a Century of Clinical, Epidemiological, and Laboratory Investigation. Heart, 91, 3-4. https://doi.org/10.1136/hrt.2004.034744

[4] Valocik, G., Kamp, O. and Visser, C.A. (2005) Three-Dimensional Echocardiography in Mitral Valve Disease. European Journal of Echocardiography, 6, 443-454. https://doi.org/10.1016/j.euje.2005.02.007

[5] Gottdiener, J.S., Bednarz, J., Devereux, R., Gardin, J., Klein, A., Manning, W., et al. (2004) American Society of Echocardiography Recommendations for Use of Echocardiography in Clinical Trials a Report from the American Society of Echocardiography's Guidelines and Standards Committee and the Task Force on Echocardiography in Clinical Trials. Journal of the American Society of Echocardiography, 17, 1086-1119. https://doi.org/10.1016/S0894-7317(04)00675-3

[6] Kılıcgedik, A., Kahveci, G., Seyfeddin Gurbuz, A., Yucel Karabay, C., Guler, A., Cagan Efe, S., Moe Aung, S., Arslantas, U., Demir, S., Akin Izgi, I. and Kirma, C. (2017) Papillary Muscle Free Strain in Patients with Severe Degenerative and Functional Mitral Regurgitation. Arquivos Brasileiros de Cardiologia, 108, 339-346. https://doi.org/10.5935/abc.20170035

[7] Gash, A.K., Carabello, B.A., Cepin, D. and Spann, J.F. (1983) Left Ventricular Ejection Performance and Systolic Muscle Function in Patients with Mitral Stenosis. Circulation, 67, 148-154. https://doi.org/10.1161/01.CIR.67.1.148

[8] Ozdemir, K., Altunkeser, B.B., Gok, H., Içli, A. and Temizhan, A. (2002) Analysis of the Myocardial Velocities in Patients with Mitral Stenosis. Journal of the American Society of Echocardiography, 15, 1472-1478. 
https://doi.org/10.1067/mje.2002.128645

[9] Doğan, S., Aydın, M., Gursurer, M., et al. (2006) Prediction of Subclinical Left Ventricular Dysfunction with Strain Rate Imaging in Patients with Mild to Moderate Rheumatic Mitral Stenosis. Journal of the American Society of Echocardiography, 19, 243-248. https://doi.org/10.1016/j.echo.2005.09.014

[10] Simsek, Z., Karakelleoglu, S., Gundugdu, F., et al. (2010) Evaluation of Left Ventriculat Function with Strain/Strain Rate Imaging in Patients with Rheumatic Mitral Stenosis. Anadolu Kardiyoloji Dergisi, 10, 328-333. https://doi.org/10.5152/akd.2010.091

[11] Marwick, T.H. (2006) Measurement of Strain and Strain Rate by Echocardiography: Ready for Prime Time? Journal of the American College of Cardiology, 47, 1313 1317. https://doi.org/10.1016/j.jacc.2005.11.063

[12] Lee, Y.S. and Lee, C.P. (1990) Ultrastructral Pathological Study of Left Ventricular Myocardium in Patients with Isolated Rheumatic Mitral Stenosis with Normal or Abnormal Left Ventricular Function. Japanese Heart Journal, 31, 435-448.

[13] Younan, H. (2015) Role of Two-Dimensional Strain and Strain Rate Imaging in Assessment of Left Ventricular Systolic Function in Patients with Rheumatic Mitral Stenosis and Normal Ejection Fraction. The Egyptian Heart Journal, 67, 193-198. https://doi.org/10.1016/j.ehj.2014.07.003

[14] Yıldırımtürk, Ö., Helvacıoğlu, F.F., Tayyareci, Y., Yurdakul, S. and Aytekin, S. (2013) Subclinical Left Ventricular Systolic Dysfunction in Patients with Mild-toModerate Rheumatic Mitral Stenosis and Normal Left Ventricular Ejection Fraction: An Observational Study. Anadolu Kardiyoloji Dergisi, 13, 328-336.

https://doi.org/10.5152/akd.2013.100 